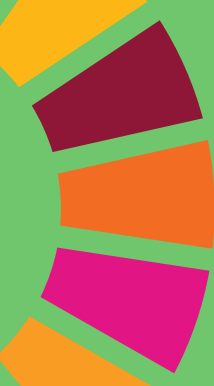

\title{
SALUD DIGITAL, SALUD GLOBAL Y ÉTICA. UNA MIRADA DESDE EL ENFOQUE DE DERECHOS HUMANOS
}

\section{DIGITAL HEALTH, GLOBAL HEALTH AND ETHICS. A LOOK FROM THE HUMAN RIGHTS APPROACH}

\author{
Celia Fernández Aller \\ Universidad Politécnica de Madrid \\ mariacelia.fernandez@upm.es
}

Fecha recepción artículo: 01/06/2020 • Fecha aprobación del artículo: 30/07/2020

\section{RESUMEN}

Cada vez es mayor el consenso en torno a la idea de que es necesaria una reflexión en torno a los principios éticos y jurídicos que deben guiar la introducción de las nuevas tecnologías (Inteligencia Artificial, Big Data, Internet de las cosas, etc.) en la salud digital. Sin ética, los usos que se hagan de la tecnología no serán compatibles con los Objetivos de Desarrollo Sostenible, ni con el bienestar de la sociedad, ni con la salud global. Además, hay que tener en cuenta que vivimos en un mundo en el que lo digital invade todo, también la salud, y en el que habrá que reinventar enfoques que sean científicamente robustos y socialmente justos.

Palabras clave: Salud digital, Tecnologías emergentes, Ética, Derechos humanos, Objetivos de Desarrollo Sostenible

\section{ABSTRACT}

There is a growing consensus on the need to reflect on the ethical and legal principles that should guide the introduction of new technologies (Artificial Intelligence, Big Data, the Internet of Things, etc.) in digital health. Without ethics, the uses made of technology will not be compatible with the Sustainable Development 
Goals, nor with the well-being of society, nor with global health. Furthermore, we must consider that we live in a world where digital technology is invading everything, including health, and where we will have to reinvent approaches that are scientifically sound and socially fair.

Keywords: Digital health, Emerging technologies, Ethic, Human rights, Sustainable Development Goals

Celia Fernández Aller Doctora en Derecho y profesora de la ETSISI (Escuela Técnica Superior de Ingeniería en Sistemas Informáticos) de la Universidad Politécnica de Madrid (UPM).

Su línea de investigación son las interrelaciones entre las TIC y los derechos humanos, con varias publicaciones sobre brecha digital, impacto social de tecnologías emergentes, etc. Pertenece al grupo de investigación de organizaciones sostenibles (GIOS) en la UPM. Adscrita al ITD UPM, Centro de Innovación en Tecnología para el Desarrollo Humano de la Universidad Politécnica de Madrid. Pertenece a un Consejo Asesor de la Fundación Alternativas.

\section{INTRODUCCIÓN}

La revolución digital es una de las seis grandes transformaciones que los expertos consideran claves para la consecución de los Objetivos de Desarrollo Sostenible (ODS) (Sachs et al., 2020). De hecho, hay voces que reconocen que el poder de la tecnología no ha sido suficientemente reflejado en la Agenda 2030. Nos encontramos de lleno en la cuarta revolución industrial, que incorpora la ubicuidad de la tecnología digital en la vida diaria y la fusión entre los mundos físico, biológico y digital.

Es necesaria una reflexión en torno a los principios éticos y jurídicos que deben guiar la introducción de las nuevas tecnologías (Inteligencia Artificial, Big Data, Internet de las cosas, etc.) en la salud digital. Sin ética, los usos que se hagan de la tecnología no serán compatibles con los ODS, ni con el bienestar de la sociedad, ni con la salud global.

Vivimos en un mundo en el que lo digital invade todo, también la salud, y en el que habrá que reinventar enfoques que sean científicamente robustos y socialmente justos.

Un informe publicado recientemente por el Relator Especial de Naciones Unidas sobre extrema pobreza y derechos humanos $(2019$, p5.) advierte sobre el riesgo de tropezar como zombies en una distopía de bienestar digital donde Big Tech ha sido un impulsor de la creciente desigualdad y ha facilitado la creación de una vasta subclase digital. El informe proporciona muchos ejemplos bien documentados en diferentes países sobre cómo las tecnologías inteligentes deshumanizadas están creando barreras para acceder a una gama de derechos sociales para quienes carecen de acceso a Internet y habilidades digitales.

\section{TECNOLOGÍA EN LA SALUD DIGITAL}

Vamos a analizar algunas tecnologías que vienen siendo utilizadas, incluso antes de la crisis del COVID-19, en el ámbito de la salud. En primer lugar, la Inteligencia Artificial (IA), que ha acompañado al Internet de las cosas en Medicina (loMT) en algunos países como China, por ejemplo, en el contexto del diagnóstico médico, utilizando tomografías computarizadas para diagnosticar casos de COVID-19. Tanto la IA como el Big Data han tenido impactos adicionales durante la pandemia, en logística para localizar y distribuir los suministros médicos en el país, así como para hacer seguimiento de la producción y la demanda (Becky McCall, 2020). 
En este momento, el papel del Big Data resulta esencial también en el ámbito de la salud. Con dicho término se hace referencia al "conjunto de tecnologías, algoritmos y sistemas empleados para recolectar datos a una escala y variedad no alcanzada hasta ahora y a la extracción de información de valor mediante sistemas analíticos avanzados soportados por computación en paralelo" (Agencia Española de Protección de Datos [AEPD] y Asociación Española para el Fomento de la Seguridad de la Información [ISMS Forum Spain], s/f)². Con base en este conocimiento generado se podrán tomar mejores decisiones.

El Big Data puede llegar a suponer, si su despliegue no respeta ciertos límites ético-jurídicos, un cambio de paradigma en diferentes campos del conocimiento. Ese cambio de paradigma se centra en el abandono de la causalidad como criterio central y su sustitución por la correlación. Esto puede generar dificultades en la explicabilidad de las decisiones que se tomen basadas en algoritmos de Big Data, que están siendo utilizados para los usos más diversos, como el mejor conocimiento del cliente, del mercado, la personalización de productos o servicios mediante la creación de perfiles, la mejora en la toma de decisiones médicas, la previsión del comportamiento de un determinado tratamiento o la monetización.

Centrándonos en el caso del Big Data para la elaboración de perfiles, existen riesgos como la reidentificación (conseguir identificar al sujeto a pesar de la anonimización), las consecuencias discriminatorias (que afectan a individuos) o las correlaciones espurias (conclusiones que, aparentemente están relacionadas, pero que en realidad no tienen ninguna relación, lo cual hace necesario reforzar la explicabilidad de los algoritmos tal y como prevé el artículo 13 del Reglamento Europeo de Protección de Datos).

Otra tecnología que tendrá impactos en la salud es el despliegue masivo del 5G, que permitirá nuevos servicios y aplicaciones relacionados con la energía, el transporte, la seguridad, y en general todos aquellos asociados al Internet de las Cosas, ya sean para uso empresarial o ciudadano. Se hace necesario garantizar que estos servicios, especialmente aquellos más estratégicos y críticos, como los de salud, cumplan con los requisitos necesarios en lo que respecta a la seguridad, fiabilidad, privacidad, y derechos de usuarios en general. Estos requisitos de seguridad habrán de ser cumplidos también por las aplicaciones, servicios y redes "virtualizadas" que hagan uso de las innovadoras capacidades de compartición de recursos que ofrece la tecnología 5G.

La telemedicina se ha ido poco a poco extendiendo dando soporte a lugares remotos, como por ejemplo la experiencia de Enlace Hispanoamericano de Salud (EHAS) en zonas remotas de países en desarrollo². Esta experiencia, y otras similares, permite monitorizar pacientes de forma remota, $y$ han traído primeras experiencias de telediagnóstico mediante técnicas de tele-estestoscopía, tele-ecografía o tele-microscopía. Pero con el 5G se puede hacer mucho más. El 18 de enero de 2019, se llevó a cabo en China la primera operación en remoto, utilizando tecnología 5G, y dos meses después la primera experiencia de neurocirugía en remoto. La realidad virtual, unida a las capacidades tecnológicas del 5G, permite a un médico operar a miles de kilómetros.

El 5G permitirá el avance del Internet de las cosas, el vehículo autónomo, el uso intensivo de big data e inteligencia artificial. Pero estas tecnologías se basan en el tratamiento de enormes cantidades de datos personales. Cualquier nivel de digitalización trae consigo la necesidad de utilización masiva de información de carácter personal. Por este motivo, el derecho a la protección de datos personales es hoy día clave. De hecho, la privacidad figura como el asunto que más preocupación suscita entre los expertos de la Ética

\footnotetext{
${ }^{1}$ Para más información consultar el Código de buenas prácticas en protección de datos para proyectos Big Data, disponible en: https:// www.aepd.es/sites/default/files/2019-09/guia-codigo-de-buenas-practicas-proyectos-de-big-data.pdf

2 Para más información consultar EHAS - Enlace Hispano Americano de Salud (www.ehas.org).
} 
Informática (Stahl, Mittelstadt y Timmermans, 2016).

El sistema de salud tiende, progresivamente, a estar basado en datos y conducido por algoritmos. De esta forma, habrá cada vez más una continua circulación de datos, muchos de carácter personal (genéticos, de comportamiento, biométricos, salud, entre otros). Esta información se recogerá de diversas fuentes (wearables, imágenes, redes sociales, instrumentos quirúrgicos, información geo-espacial) que manejan los pacientes, personal clínico, seguros y sistemas de salud. La Inteligencia Artificial podría dar consejos a las personas antes de que los problemas se conviertan en significativos, de forma que la demanda de servicios médicos podría ser predicha, evitándose muertes.

Las oportunidades no surgen a partir de la tecnología per se, sino a partir de la habilidad para utilizar los sistemas, a partir de las formas en las que los sistemas de salud proveen los servicios mediante el acoplamiento, desacoplamiento y re-acoplamiento de las diferentes partes del sistema de salud (Floridi, 2019):

- Acoplamiento: los pacientes y sus datos están tan interrelacionados que los pacientes son sus perfiles genéricos, sus resultados de los análisis de sangre, su información personal sobre alergias, etc. Lo que las normas de protección de datos denominan sujetos de los datos, se han convertido en "pacientes de los datos".

- Desacoplamiento: la presencia del proveedor de servicio de salud y la localización del paciente se han desacoplado, por ejemplo, por la introducción de las consultas on line.

- Re-acoplamiento: la investigación y la práctica se habían separado pero, en el mundo digital, son una misma cosa de nuevo.

En este sentido, un asunto urgente que habrá de afrontar en la Ética de las Tecnologías de la Información y las Comunicaciones (TIC) es el de la justicia algorítmica, de modo que la utilización de los algoritmos de decisión sean explicables, y pueda verificarse que las consecuencias de las opciones que tomen son justas (si me deniegan una ayuda social, por ejemplo, he de poder conocer los motivos). Son conocidas las consecuencias del uso de la analítica de la pobreza para evaluar la situación social de las personas, su perfil ante la policía, las condenas penales (Eusbanks, 2019). La idea central detrás de estos proyectos es que la pobreza es, en esencia, un problema de ingeniería de sistemas. Pero se ha demostrado que la utilización de IA para controlar la gestión de las ayudas sociales en Estados Unidos no ha traído más justicia en el reparto de las ayudas, sino al contrario.

\section{ANÁLISIS DEL MARCO ÉTICO-JURÍDICO}

Nuestra sociedad es cada vez más tecnodependiente, y de forma especial depende de las Tecnologías de la Información y las Comunicaciones (TIC). Un fallo en las TIC puede dar lugar a consecuencias e impactos serios en la vida de las personas. Por este motivo, y por la especificidad de los conocimientos, surge la necesidad de la responsabilidad ética. La Ética es una disciplina esencial, pero no como algo instrumental y dedicado a resolver problemas concretos, sino como aporte en el diseño de valores y principios que nos orienten en nuestra práctica. Estos valores son, fundamentalmente, el conocimiento riguroso, el beneficio a la humanidad, la eficiencia, la sensatez, la honestidad y la responsabilidad ante la naturaleza.

Tal y como propone Carissa Véliz (2019), los códigos éticos son necesarios para recoger principios éticos consensuados aplicables a las diferentes áreas profesionales, así como para recoger buenas prácticas. El mundo digital tiene una urgente necesidad de códigos, de forma análoga al Código de Nuremberg, a la 
Declaración de Ginebra, al Informe Belmont o a la Declaración de Helsinki, que han definido las políticas de investigación y médicas alrededor del mundo a pesar de su naturaleza no vinculante.

No puede pensarse que los textos legales, como el Reglamento General de Protección de Datos (RGPD), son suficientes para resolver los problemas éticos que aparecen en relación a la salud digital. Esta norma se refiere exclusivamente a los problemas generados con los datos personales, pero no tiene soluciones para problemas como la programación de decisiones éticas en los vehículos autónomos o los dilemas éticos de las tecnologías emergentes.

Por otro lado, las leyes son estrechas en cuanto a su alcance, establecen requisitos mínimos de comportamiento para instituciones sociales. La Ética va más allá, identificando asuntos morales que tratan de reflejar la sociedad en la que nos gustaría vivir. La Ética sirve para rellenar lagunas legales, para ir dibujando el camino de las decisiones cuando los problemas que ocasiona la tecnología son nuevos.

Es cierto que grandes empresas tecnológicas como Google ${ }^{3}$ han hecho esfuerzos por dotarse de códigos éticos, como el relativo a la Inteligencia Artificial. Estos esfuerzos de las empresas por pensar en los asuntos éticos y comprometerse a ciertas pautas son muy deseables. Pero el interés privado no puede marcar el rumbo de los consensos éticos, al faltarle la imparcialidad.

Tal y como ha señalado recientemente Nemitz (2018), debemos estar muy vigilantes con las actividades de los "temibles 5", que son quienes moldean nuestra experiencia con las tecnologías digitales, incluida la IA: Google, Facebook, Microsoft, Apple y Amazon. Estas corporaciones son extremadamente ricas, lo que les garantiza acceso desproporcionado a legisladores y gobiernos. Además, financian todo tipo de actividades, incluidas la ciencia y la investigación. Estas empresas están presentes en todos los campos, tanto político, como de la sociedad civil, ciencia, periodismo, negocios, lo que les permite ganarse la simpatía en torno a los asuntos que les preocupan. Cualquier análisis crítico debe comenzar por la comprensión de esta acumulación de poder tecnológico, económico y político en las manos de las referidas empresas, que lideran el desarrollo tecnológico de la IA y su transformación en servicios de interés comercial (Nemitz, 2018).

Las formulaciones éticas de las empresas suelen ser muy amplias y se produce en ocasiones una selección del código ético por parte de las mismas que se adapta a sus necesidades sin un proceso de consultas y acuerdo entre las partes interesadas. En otras palabras, habrían de evitarse los peligros de los que alerta Floridi (2019): a) Ethics shopping, que supone que una orga nización elija, entre las muchas iniciativas que hay de códigos éticos muy dispersos, el que mejor se adapte a su forma de hacer, justificando así sus intenciones, poco coherentes con la ética en ocasiones. b) Ethics dumping, que consiste en la conducta de exportar prácticas no éticas a países donde hay más laxitud o diferencia de criterios. c) Ethics lobbying, o la práctica de algunos actores privados de usar autorregulación en temas como la ética de la Inteligencia Artificial para hacer lobbying en contra de la introducción de normas con fuerza jurídica, sometidas estas últimas a mecanismos más exigentes en caso de incumplimiento. d) Bluewashing, como concepto proveniente de la ética de la Ecología -greenwashing- (Delmas and Burbano 2011), que es la mala práctica de una organización pública o privada que busca aparecer socialmente como más verde, sostenible y comprometida de lo que en realidad es.

Por otro lado, hay que mencionar que existen demasiadas propuestas de principios éticos de aplicación en el

\footnotetext{
${ }_{3}^{3}$ Para más información consultar Pichai, S. "IA at Google: our principles". Google (7 June 2018). Disponible en: https://go.nature. com/2LJvzhY
} 
campo tecnológico, descoordinadas entre sí: Association for Computing Machinery (ACM), Institute of Electrical and Electronics Engineers (IEEE), Principios de Asilomar para la Inteligencia Artificial', el Contrato de la web de la WWW Foundation, Naciones Unidas, a través de su Relator sobre el derecho a la privacidad's, Unión Europea, Telefónica, Internet Society, Global Network Initiative ${ }^{6}$ (GNI), Ranking Digital Rights (RDR), por citar algunas.

Por otro lado, cualquier marco ético debe descansar en los derechos humanos, como valores éticos esenciales consensuados al máximo nivel. Además, los derechos humanos están en la base de todos y cada uno de los ODS.

No podemos olvidarnos de que "tenemos los mismos derechos on line y off line" tal y como ha recordado el Consejo de Derechos Humanos de Naciones Unidas (2012). Esto quiere decir que nuestra sociedad tiene pendiente conseguir un reconocimiento y respeto a los derechos humanos en el mundo virtual, que se asemeje al mundo analógico. No podemos pensar que el hecho de que una conducta se desarrolle en internet la haga caer en un limbo ético jurídico.

En este sentido, urge una adaptación y aplicación honesta del marco del enfoque de derechos humanos a la revolución tecnológica que está en marcha. El enfoque de derechos humanos está ampliamente aceptado en el ámbito internacional, y cada vez más expertos resaltan la importancia de los derechos humanos para analizar el impacto social de las tecnologías emergentes.

Esto tiene dos ventajas: Por un lado, el enfoque promueve el cumplimiento de la ley interna e internacional. Por otro lado, el mismo contenido de los derechos humanos sirve como un espejo frente al que contrastar los impactos de las nuevas tecnologías.

Adoptar el enfoque de derechos humanos implica incluir los derechos humanos en todos los aspectos de las políticas y toma de decisión. Algunos aspectos clave del enfoque son:

- Promover la transparencia en los procesos de gobernabilidad. Los gobiernos debieran tener en cuenta el impacto de la gobernanza de las tecnologías en los derechos humanos.

- Asegurar la rendición de cuentas, a través de un marco regulatorio que distinga claramente las obligaciones de los Estados y de los actores no estatales (entre los que estarían incluidas empresas, entre otros), que tienen responsabilidades definidas en los marcos legislativos gubernamentales. Esto debería promover el respeto de las empresas por los derechos humanos, de acuerdo con los Principios de Empresa y Derechos Humanos de Naciones Unidas.

- Asegurar que la aplicación de las nuevas tecnologías respeta el principio de no discriminación. Esto implica que cualquier desarrollo tecnológico debe incluir a las personas con discapacidad, por lo que los asuntos de accesibilidad electrónica cobran toda la relevancia. Quizá sea necesario preguntarse además cómo podría ayudar la IA a sectores desfavorecidos de la población (o incluso perjudicar si no se ponen medidas). El riesgo es crear una nueva brecha de IA, que se superpondría a la ya preocupante de por sí brecha digital que se mantiene hoy día a nivel mundial?.

\footnotetext{
${ }^{4}$ Para más información consultar en https://futureoflife.org/IA-principles/

${ }^{5}$ Para más información consultar en https://www.ohchr.org/EN/Issues/Privacy/SR/Pages/SRPrivacylndex.aspx

${ }^{6}$ Para más información consultar en https://globalnetworkinitiative.org/about-gni/

${ }^{7}$ ITU (2019) Bridging the digital divide.
} 
- El enfoque de derechos requiere aproximaciones participativas, en las que se tomen en cuenta las voces de todos los actores.

- La construcción de capacidades se debe producir desde la comprensión de la comunidad de los impactos que las tecnologías han de tener en sus vidas.

Se producen intersecciones muy claras entre los derechos humanos y las tecnologías. Estas pueden promover o restringir aquellos, y a veces ofrecen ambas posibilidades a la vez (Australian Human Rights Commission, 2019, p.17):

- "Derecho a la equidad y no discriminación". Las aplicaciones que utilizan la IA, y especialmente el machine-learning, deben ser "entrenadas" usando datos. Cuando esos datos incorporan injusticias, como la discriminación, esto puede replicarse en la nueva aplicación (Kusner y Loftus, 2020). Cuando los datos de capacitación se recogen y utilizan bien, las nuevas tecnologías como la IA pueden permitir una mejor prestación de servicios, especialmente para los grupos vulnerables. El acceso desigual a nuevas tecnologías críticas puede exacerbar las desigualdades, especialmente cuando el acceso se ve afectado por factores como la situación socioeconómica, la discapacidad, la edad o la ubicación geográfica.

- "Libertad de expresión. Las nuevas tecnologías pueden permitir una vigilancia a gran escala en línea y en el mundo físico, lo que puede disuadir a las personas de compartir legítimamente sus opiniones.

- "Derecho a beneficiarse de los progresos científicos". Las nuevas tecnologías pueden mejorar el disfrute de derechos humanos como el acceso a la alimentación, la salud y la educación. Garantizar la accesibilidad en todos los sectores de la comunidad puede ser difícil.

- "Accesibilidad". Las nuevas tecnologías pueden proporcionar nuevas formas de prestar servicios, aumentando así la accesibilidad para las personas con discapacidad y otras personas. La reducción del costo de los servicios gracias a la asequibilidad de las nuevas tecnologías puede promover la igualdad para las personas con discapacidad, asegurando que la realización progresiva se logre más rápidamente y que los ajustes razonables sean más asequibles. Las nuevas tecnologías pueden aumentar las barreras para las personas con discapacidad si se utilizan en productos y servicios de manera no accesible.

- "Protección de la comunidad y la seguridad nacional". Las nuevas tecnologías pueden aumentar la capacidad de los gobiernos para identificar las amenazas a la seguridad nacional. El uso de esas tecnologías con fines de vigilancia puede ser excesivamente amplio y, sin las salvaguardias apropiadas, puede afectar de manera irrazonable a la privacidad y la reputación de personas inocentes.

- "Derecho a la privacidad". La facilidad de reunir y utilizar información personal mediante nuevas tecnologías, como el reconocimiento facial, puede limitar el derecho a la intimidad y a la privacidad. Los datos personales pueden fluir fácil y rápidamente. Esto puede dificultar la regulación y la aplicación de la privacidad. Puede ser difícil "corregir" o eliminar la información personal una vez comunicada. La facilidad de comunicar y distorsionar la información personal (por ejemplo, a través de "falsificaciones profundas") puede conducir a daños de reputación y otros perjuicios.

- "Derecho a la educación". Las nuevas tecnologías pueden mejorar la disponibilidad y accesibilidad de la educación. La falta de acceso a la tecnología puede exacerbar la desigualdad, basada en factores como la edad, la discapacidad, la condición de indígena y la ubicación rural o remota. 
- "Acceso a la información y seguridad de los niños". Los entornos en línea brindan a los niños la oportunidad de acceder a una gran cantidad de información, pero también plantean problemas para su bienestar. Las nuevas tecnologías crean diferentes entornos para el acoso y la intimidación que a veces son difíciles de moderar. La tecnología digital también puede facilitar la explotación de los niños.

Parece razonable que se comience a hablar incluso de una responsabilidad social tecnológica, concepto que requerirá mayor atención a partir de ahora.

\subsection{UN CASO CONCRETO: RETOS ÉTICOS Y JURÍDICOS DE LA IA EN RELACIÓN AL COVID-19}

Un asunto de salud global que está siendo objeto de reflexión hoy día es la utilización de aplicaciones TIC para hacer seguimiento del COVID-19. Indudablemente, estas aplicaciones son lícitas, siempre y cuando se tomen algunas precauciones básicas en protección de datos, como la solicitud de consentimiento y la información suficiente al interesado. Además, desde el punto de vista de la privacidad, las aplicaciones han de incorporar medidas de seguridad suficientes.

Habrán de vigilarse los posibles sesgos del modelo de IA que se utilice, y el principio de no discriminación: Las soluciones tecnológicas deben ajustarse a este principio, impidiéndose que las decisiones que se tomen generen brechas digitales que dejen al margen de los avances a minorías y personas vulnerables (Eusbanks, 2019), como personas con discapacidad, mayores, en situación de desventaja económica, infancia, etc.

Las aplicaciones deben estar disponibles también en países donde el nivel de desarrollo tecnológico no sea el mismo que en países desarrollados, de otro modo, los principios de solidaridad y justicia se conculcarían.

Expertos en privacidad alertan de que, en cierto sentido, mi valor como persona está hoy día representado por mi vida digital (cómo me ven los demás, cuántos seguidores tengo en redes sociales, qué dicen los bancos sobre mi solvencia, qué coberturas merezco frente a las aseguradoras, o, en el caso que nos ocupa, si soy o no persona de riesgo en relación al COVID-19-teniendo en cuenta con quién me he relacionado, cuál es mi historial médico, etc.). Es necesario devolver la confianza en el uso que se está haciendo de los datos personales, desarrollando tecnologías robustas (que sitúen los asuntos de seguridadentre los más prioritarios) y con propósito ético.

Puede ser muy valioso en este caso extrapolar las DIRECTRICES ÉTICAS para una IA fiable, que ha desarrollado el Grupo de expertos de alto nivel sobre inteligencia artificial de la Unión Europea en 2019 (Comisión Europea, 2019).

- Desarrollar, desplegar y utilizar los sistemas respetando los principios éticos de: respeto de la autonomía humana, prevención del daño, equidad y explicabilidad. Reconocer y abordar las tensiones que pueden surgir entre estos principios.

${ }^{8}$ Para más información ver Vid. The Cloud Security Alliance (CSA) Big Data Working Group (BIG DATAWG) has come up with 100 best practices to enhance the security and privacy of Big Data: https://docs.google.com/document/d/1FqeHIA53sliNS3sd3ECy2hwyJuOU JDZT71zUs-02nX4/edit

The top 10 best practice concerning security are: 1 . Authorize access to files by predefined security policy

2. Protect data by data encryption while at rest 3. Implement Policy Based Encryption System (PBES)

4. Use antivirus and malware protection systems at endpoints 5. Use Big Data analytics to detect anomalous connections to cluster 6. Implement privacy preserving analytics 7. Consider use of partial homomorphic encryption schemes 8. Implement fine grained access controls 9. Provide timely access to audit information 10. Provide infrastructure authentication mechanisms 
- Prestar una atención especial a las situaciones que afecten a los grupos más vulnerables, como los niños, las personas con discapacidad y otras que se hayan visto históricamente desfavorecidas o que se encuentren en riesgo de exclusión, así como a las situaciones caracterizadas por asimetrías de poder o de información.

- Reconocer y tener presente que, pese a que aportan beneficios sustanciales a las personas y a la sociedad, los sistemas de IA también entrañan determinados riesgos y pueden tener efectos negativos, algunos de los cuales pueden resultar difíciles de prever, identificar o medir (por ejemplo, sobre la democracia, el estado de Derecho y la justicia distributiva, o sobre la propia mente humana).

- Garantizar que el desarrollo, despliegue y utilización de los sistemas cumplan los requisitos para una IA fiable: 1) acción y supervisión humanas, 2) solidez técnica y seguridad, 3) gestión de la privacidad y de los datos, 4) transparencia, 5) diversidad, no discriminación y equidad, 6) bienestar ambiental y social, y 7) rendición de cuentas.

- Para garantizar el cumplimiento de estos requisitos, se deberá estudiar la posibilidad de emplear tanto métodos técnicos como no técnicos.

- Impulsar la investigación y la innovación.

- Comunicar información a las partes interesadas, de un modo claro y proactivo, sobre las capacidades y limitaciones de los sistemas, posibilitando el establecimiento de expectativas realistas, así como sobre el modo en que se cumplen los requisitos. Ser transparentes acerca del hecho de que se está trabajando con un sistema.

- Facilitar la trazabilidad y la auditabilidad de los sistemas, especialmente en contextos o situaciones críticos.

- Adoptar una evaluación de la fiabilidad al desarrollar, desplegar o utilizar sistemas, y adaptarla al caso de uso específico en el que se aplique dicho sistema.

- Tener presente que este tipo de listas de evaluación nunca pueden ser exhaustivas. Garantizar la fiabilidad no consiste en marcar casillas de verificación, sino en identificar y aplicar constantemente requisitos, evaluar soluciones y asegurar mejores resultados a lo largo de todo el ciclo de vida del sistema de IA, implicando a las partes interesadas en el proceso.

\section{CONCLUSIONES}

Se torna urgente la aceptación de una nueva disciplina, la Ciberética, como ciencia aplicada que se sitúa entre las Ciencias de la Computación y la Ética. Un ámbito clave en la Ciberética es precisamente el objeto de este estudio: la compatibilidad entre los derechos y la Inteligencia artificial.

Un asunto clave de esta disciplina es el contexto de la gobernanza de los datos personales, especialmente los referentes a la salud, que ha sufrido un cambio radical con el desarrollo de las tecnologías de IA. La gobernanza de los datos tiene un peso muy importante en cualquier proyecto de ingeniería, en especial si los datos son personales. La rapidez con la que se tienen que almacenar la información unida a la extensión en su tipología, hace muy difícil que los procesos de verificación y calidad utilizados hasta ahora sean totalmente eficaces, por lo que es necesario crear nuevas metodologías y herramientas adecuadas. Se calcula que si 
utilizásemos DVD para mover los datos que se utilizan a nivel global necesitaríamos fletar 16 millones de Jumbos, datos de 2015 (Kuner, Cate, Millard y Svantesson, 2012). La Unión Europea acaba de publicar una comunicación sobre la estrategia europea de los datos`, en la que se plantea precisamente la creación de un espacio europeo de datos de salud.

A nivel global se constata una falta de gobernanza: hay instituciones nacionales, y algunas supranacionales, pero se evidencia con demasiada frecuencia la imposibilidad de conseguir instituciones sólidas que actúen a nivel mundial cuando determinados actores vulneran la privacidad, la libertad de elección política, la dignidad de las personas ${ }^{10}$.

Sin privacidad, ni internet ni las tecnologías de tratamiento de información personal pueden funcionar en el marco de nuestras sociedades democráticas. Es de tal importancia el tema que planteamos que se está hablando de la necesidad de un nuevo contrato social, en el que se pacten todos los aspectos que hoy día carecen de regulación y, lo que es peor, de consenso.

Existe la necesidad de una investigación independiente y científicamente rigurosa en el campo académico, con una dimensión empírica hasta ahora bastante débil. Entre las líneas de investigación y desarrollo que habría que fomentar y subvencionar tendrían que ser prioritarias las relativas a los principios éticos, códigos de conducta y legislación, aunque también se requieren herramientas para aplicar estos códigos de forma práctica. El problema de mitigar los riesgos de la IA en la salud global puede venir asimismo de la mano de herramientas que incorporen técnicas IA. Es necesario un esfuerzo coordinado multidisciplinar que implique a investigadores, innovadores, ciudadanos, legisladores, políticos, desarrolladores, entre otros, para crear y evaluar estas herramientas. La multidisciplinariedad es esencial para dar pleno sentido y desde diferentes perspectivas a los conceptos de explicabilidad, transparencia, etc., para comprender la complejidad del comportamiento humano y los impactos que en él pueden tener las tecnologías IA. Además hay que garantizar que no sólo se protege la pluralidad de valores de los profesionales y productores sino de la sociedad en general. La ética tiene que estar embebida en el proceso de diseño, desarrollo, despliegue y uso de la tecnología inteligente. Los principios éticos tienen que traducirse en protocolos de diseño, desarrollo, despliegue y uso. Se requieren herramientas metodológicas y técnicas específicas. No se trata de que estas herramientas reemplacen la legislación y los manuales de ética y buenas prácticas, sino de que respalden su implementación. La investigación académica, la autorregulación del sector privado y la legislación son acciones necesarias y complementarias.

Los estudios de impacto ético son altamente recomendables en las aplicaciones de IA que utilicen datos personales de salud. Así mismo, son exigibles por ley los estudios de impacto en la privacidad. Y por último, comienzan a utilizarse los estudios de impacto algorítmico, como requisitos previos a la utilización de sistemas de IA que puedan impactar negativamente en los derechos humanos, entre ellos el derecho a la salud.

\footnotetext{
${ }^{9}$ Para más información consultar en Bruselas, 19.2.2020 COM(2020) 66 final Comunicación de la Comisión al Parlamento Europeo, al Consejo, al Comité Económico y Social Europeo y al Comité de las Regiones: Una Estrategia Europea de Datos.

${ }^{10}$ Para más información se puede consultar: Facebook's Role in Brexit., disponible en: https://www.ted.com/talks/carole_cadwalladr_ facebook_s_role_in_brexit_and_the_threat_to_democracy?language=en
} 


\section{REFERENCIAS BIBLIOGRÁFICAS}

- Agencia Española de Protección de Datos y Asociación Española para el Fomento de la Seguridad de la Información (s/f) Código de buenas prácticas en protección de datos para proyectos Big Data.

- Australian Human Rights Commission (2019) Human Rights and Technology. Discussion Paper, p. 17.

- BBVA (2020) El trabajo en la era de los datos. Recuperado de https://www.bbvaopenmind.com/libros/eltrabajo-en-la-era-de-los-datos/

- Becky McCall (2020). The Lancet. Elsevier

- Cloud Security Alliance (CSA) Big Data Working Group (BIG DATAWG) has come up with 100 best practices to enhance the security and privacy of Big Data: https://docs.google.com/document/d/1FqeHIA 53sliNS3sd3ECy2hwyJuOUJDZT71zUs-02nX4/edit

- Comisión Australiana de Derechos Humanos (2019) Human Rights and Technology. Discussion paper.

- Comisión Europea (2019). Directrices éticas para una IE fiable. DOI 10.2759/14078

- Eusbanks, V (2019). La automatización de los prejuicios. Investigación y Ciencia, 508.

- Floridi, L (2019). Translating principles into Practices of Digital Ethics: Five Risks of Being Unethical. Philosophy and Technology, 32, 185-193.

- ITU (2019) Bridging the digital divide.

- Kuner, C., Cate, F. H, Millard, C., y Svantesson, D. J. B. (2012). The challenge of 'big data' for data protection. International Data Privacy Law, 2 (2). DOI: 10.1093/idpl/ips003

- Kusner, M. J. y Loftus, J. R. (6 de febrero 2020) "The long road to fairer algorithms" Nature. 578.

- Morley, J. y Floridi1, L. (2019). How to design a governable digital health ecosystem. DOI: 10.13140/ RG.2.2.28320.74244/1

- Nemitz, Paul. Democracy and Technology in the Age of Artificial Intelligence (August 18, 2018). DOI 10.1098/RSTA.2018.0089 - Royal Society Philosophical Transactions. Available at SSRN: https://ssrn.com/ abstract $=3234336$

- Pichai, S. (7 June 2018). IA at Google: our principles. [Google]. Recuperado de: https://go.nature. com/2LJvzhY

- Relator Especial de Naciones Unidas sobre pobreza extrema y derechos humanos. Informe del Relator Especial. A/74/493 General Assembly Distr.: General 11. Promotion and protection of human rights: human rights questions, including alternative approaches for improving the effective enjoyment of human rights and fundamental freedoms (11 de octubre de 2019). Transcripción. Recuperado de https:// undocs.org/pdf?symbol=en/A/74/493

- Sachs, J.D, Schimidt-Traub, G, Mazzucato, M, Messner, D, Nakicenovic, N, Rockstrom (2020). "Six Transformations". Nature Sustainability.

- Stahl, B. C., Mittelstadt, B., y Timmermans, J. (2016). The Ethics of Computing. ACM Computing Surveys. DOI: 10.1145/2871196, p.29

- Véliz, C. Three things digital ethics can learn from medical ethics (Agosto de 2019). Nature Electronics. DOI 10.1038/s41928-019-0294-2 